\title{
TGF- $\beta$ Induces Up-Regulation of Chondroitin Sulfate Synthase 1 (CHSY1) in Nucleus Pulposus Cells Through MAPK Signaling
}

Bo Hu Changgui Shi Ye Tian Ying Zhang Chen Xu Huajiang Chen Peng Cao Wen Yuan

Department of Spinal Surgery, Changzheng Hospital, Second Military Medical University, Shanghai, China

\section{Key Word}

Nucleus pulposus $\bullet$ CHSY1 1 TGF- $\beta \cdot$ MAPK

\begin{abstract}
Background/Aims: Chondroitin sulfate synthase 1 (CHSY1) is a glycosyltransferases involved in the biosynthesis of chondroitin and dermatan sulfate glycosaminoglycan (GAG). TGF- $\beta$ can stimulate sulfated GAG production in nucleus pulposus cells; however, the underlying mechanisms are poorly understood. Methods: CHSY1 expression was examined in rat nucleus pulposus treated with TGF- $\beta$ using real-time PCR and Western blot analysis. Lentiviral knockdown was performed to determine the downstream effectors of TGF- $\beta$ and to measure the effect of c-Jun and Sp1 on TGF- $\beta$ mediated CHSY1 promoter activity and CHSY1 expression. Results: TGF- $\beta$ increased CHSY1 expression and promoter activity in the nucleus pulposus partially through activation of canonical Smad signaling pathway. Knockdown of c-Jun and Sp1 decreased CHSY1 promoter activity, CHSY1 expression and sGAG accumulation induced by TGF- $\beta$. Furthermore, we found that TGF- $\beta$-induced expression of CHSY1 was mediated through the activation of MAPK signaling. Moreover, we showed that silencing CHSY1 decreased sGAG accumulation in nucleus pulposus cells induced by TGF- $\beta$. Conclusion: Our results suggest that TGF- $\beta$ induced CHSY1 expression in the nucleus pulposus through the activation of MAPK signaling.
\end{abstract}

Copyright (c) 2015 S. Karger AG, Basel

\section{Introduction}

Intervertebral discs are joints between vertebrae and function to buffer biomechanical loads as well as to provide flexibility of the otherwise rigid spine. The discs consist of a variety

B. Hu and C. Shi equally contributed to this paper.

Peng Cao and Wen Yuan

KARGER 125
Department of Spinal Surgery, Changzheng Hospital, Second Military Medical

University, No. 415 Feng Yang Rd, Shanghai 200003, (China)

E-Mail mdpinocao@163.com and yuanwenspine@126.com 
of different tissues, among which the highly hydrated nucleus pulposus (NP) allows the discs to equalize hydraulic pressure under compressive loads [1]. The NP is a heterogeneous structure containing a substantially high percentage of proteoglycans and type II collagen. Particularly, higher expression of aggrecan and synthesis of proteoglycan distinguish NP cells from other types of cells within the discs [2]. Aggrecan is a protein heavily modified by large carbohydrates, with glycosaminoglycan (GAG) as the most common form in the NP cells. Because the GAG from of aggrecan molecules is highly polar and hydrophilic, it provides a robust hydrodynamic system that accommodates applied biomechanical forces $[3,4]$. Structurally, the GAG moieties are covalently linked to the core of aggrecan protein through 0 -glycocylation catalyzed by glycosyltransferases, leading to the formation of chondroitin and dermatan sulfate proteoglycans [5]. Chondroitin sulfate synthase 1 (CHSY1) is one type of glycosyltransferases involved in chondroitin and dermatan sulfate GAG biosynthesis [6]. Animals with chsy1 deficiency display multiple skeletal defects including chondrodysplasia and decreased bone density, largely due to an imbalance in chondroitin sulfation [6]. Interestingly, loss-of-function mutations at the chsy1 locus have been identified in human syndromic recessive preaxial brachydactyly, suggesting the importance of this gene product in the development of proper bone [7, 8]. Additionally, CHSY1 was also reported to co-express with aggrecan, highlighting its contribution in the production of aggrecan in NP, and thereby in the function of maintaining the intervertebral disc [9].

Given the essential roles of aggrecan in the function of the intervertebral discs, it is not surprising that the biosynthesis and sulfation of proteoglycan are closely regulated. TGF- $\beta$ signaling pathway represents one of the important regulations [10]. TGF- $\beta$ is known to induce the genes encoding of the core proteins of chondroitin sulfate proteoglycans and the enzymes regulating GAG chain deposition [11]. In the mammalian cells three TGF- $\beta$ isoforms, TGF- $\beta 1,-\beta 2$ and $-\beta 3$, have been identified. The binding of TGF- $\beta$ ligands to their cognate receptors initiates a cascade of phosphorylation events leading to the activation of SMADs and ultimately transcription of target genes. Intriguingly, the TGF- $\beta$ subtype, TGF- $\beta 3$, has been shown to not only increase the survival and viability of the cells in the intervertebral disc but also upregulate the synthesis of sulfated GAG in NP cells in organ culture [12]. The elevated production of sulfated GAG in response to TGF- $\beta$ in NP cells is likely through transcriptional activation of CHSY1 enzyme, because the chsy1 mRNA has been reported to be up regulated in mouse astrocytes treated with TGF- $\beta$ dependent on Smad2 [13]. However, the detailed mechanisms underlying CHSY1 expression and chondroitin sulfate chain synthesis in response to TGF- $\beta$ in NP cells have not been determined.

In the present study, we demonstrated that TGF- $\beta$ treatment in NP cells resulted in the up regulation of CHSY1 at both protein and RNA levels. We confirmed that transcription factors c-Jun and Sp1 directly controlled CHSY1 promoter activity in response to TGF- $\beta$, and thereby CHSY1 expression as well as SGAG accumulation. Furthermore, we found that TGF- $\beta$ induced expression of CHSY1 was mediated by the activation of MAPK signaling. Moreover, silencing of CHSY1 blunted the increase of sGAG induced by TGF- $\beta$.

\section{Materials and Methods}

\section{Isolation and treatments of nucleus pulposus cells}

Rat nucleus pulposus cells were isolated according to a method reported earlier by Risbud et al.[14]. The cells were cultured in DEME with 10\% fetal bovine serum (FBS). Nucleus pulposus cells were washed and incubated overnight before treatment with TGF- $\beta 3$ in fresh culture medium. In some experiments cells were treated with TGF- $\beta 3$ (10 ng/ml, R\&D systems, Minneapolis, MN, USA) with or without PD98059 (30 $\mu$ M, Cell Signaling Technology, Beverly, MA, USA), SKF86002 (20 $\mu$ M, Sigma, Munich, Germany), and SP600125 (30 $\mu \mathrm{M}$, Cell Signaling Technology). To confirm that the response was specific from TGF- $\beta$, NP cells were treated with SB431542 (10 $\mu \mathrm{M}$, Sigma).

\section{KARGER}




\section{Quantitative real-time RT-PCR analysis}

Total RNA was extracted from NP cells using TRIzol reagent (Invitrogen, CA, USA). RNA extracts were treated with RNase free DNase (Invitrogen) according to the manufacturer's protocol. RNA was subsequently converted to cDNA using PrimeScript RT reagent Kit. Real-time RT-PCR was carried out with SYBR@ Premix Ex Taq ${ }^{\text {TM }}$ II (TaKaRa, Otsu, Japan) on the ABI 7500 Real-Time PCR System (Applied Biosystems, Foster City, CA, USA). The sequences of primers for rat chsy1 were as follow: forward 5'-GTT ACA TGC TCA GCC GCAAG- $3^{\prime}$ and reverse $5^{\prime}$-TCA CGC GTC TGT AGC CATAC- $3^{\prime}$. GAPDH was used as internal control. The primers used to assay GAPDH were as follow: forward 5'-TTC TCT TGT GAC AAA GTG GACAT-3' and reverse 5' -GAA GGG GCG GAG ATG ATGAC-3'.

\section{Western blotting}

NP cells were harvested at indicated time point $(0.5,1,4,8$ and $24 \mathrm{~h})$ of treatment with or without TGF- $\beta$ by iced-cold lysis buffer (Cell Signaling Technology). Cell lysates were prepared in the presence of protease inhibitor cocktail (Pierce Biotechnology, Rockford, IL, USA), and protein concentration was determined using a Bicinchoninic Acid Protein assay kit (Pierce Biotechnology). Equal amounts of protein were separated on SDS-polyacrylamide gels (8-10\%) and subsequently transferred to PVDF membranes (Bio-Rad, Berkley, CA, USA). Immunolabeling was detected using Pierce ECL Western Blotting Substrate (Pierce Biotechnology). Blot intensity was determined by densitometric analysis using Image J software. Following antibodies and dilution were used: CHSY1 antibody (sc-292185, 1:200), Sp1 (sc-59, 1:200), $\beta$-actin (sc-130656, 1:1000) (Santa Cruz Biotechnology, Santa Cruz, CA, USA); Smad2 (ab33875, 1:1000), Smad3 (ab40854, 1:2000) (Abcam, Cambridge, MA, USA); and phospho-Smad2 (Ser465/467) (\#3108, 1:1000), phospho-Smad3 (Ser423/425) (\#9520, 1:1000), phospho-c-Jun (S63) (\#9261, 1:1000), c-Jun (\#9165, 1:1000), phospho-ERK1/2 (\#9101, 1:1000), anti-ERK1/2 (\#9102, 1:1000), phospho-p38 MAPK (Thr180/Tyr182) (\#9211, 1:1000), p38 MAPK (\#9212, 1:1000), phospho-JNK (T183/Y185) (\#4671, 1:1000), and JNK (\#9252, 1:1000) (Cell Signaling Technology). Horseradish peroxidase-linked goat antirabbit (sc-2004, 1:5000) antibody was used as secondary antibody.

\section{Immunofluorescence microscopy}

Cells were treated with TGF- $\beta$ for $24 \mathrm{~h}$. Fixed cells were incubated with primary antibody against CHSY1 (sc-292185, 1:100, Santa Cruz Biotechnology) overnight, followed by an Alexa fluor-488 conjugated anti-rabbit secondary antibody (Invitrogen) at room temperature for $1 \mathrm{~h}$. Cells were reacted with isotype IgG under similar conditions as a negative control. Nuclei were counterstained with DAPI (Invitrogen, USA). Images were acquired by a laser scanning confocal microscope (Olympus, Tokyo, Japan) and all images were taken at $200 \times$ and the gain was kept constant across the experimental conditions.

\section{Promoter reporter assay}

To generate CHSY1 reporter constructs, human CHSY1 promoter DNA from the -1831 to +64 regions was amplified by PCR using primers as follow: forward, 5'-GAG AAC GCG TCA AGC GAT TCT CCT GCC TCA-3' and reverse, $5^{\prime}$-GAG ACT CGA GTG TCC CCG TCC TCT TCG TAGCC-3'. The DNA fragments were subcloned into pGEM-T-easy vector (Promega, Madison, WI, USA) using T4 DNA ligase (Promega), followed by restriction digestion with Mlu I and Xho I, and ligation into the pGL3-Basic vector (Promega).

AP1 luciferase reporter plasmids (AP1-Luc) were purchased from Clontech (Mountain View, CA, USA), Sp1 luciferase reporter plasmids (Sp1-Luc) were purchased from Sabiosciences (Frederick, MD, USA). For transfection, cells were seeded to 48 -well plates at a density of $2 \times 10^{4}$ cells/well. After $24 \mathrm{~h}$, cells were transfected with $500 \mathrm{ng}$ of CHSY1 reporter plasmids, AP1 reporter plasmids, or Sp1 reporter plasmids with 500 ng pRL-TK plasmid (Promega) using Lipofectamine 2000 (Invitrogen). After another 24 h, cells were harvested for luciferase assay by a Dual-Luciferase reporter assay system (Promega).

Lentiviral particle production and viral transduction

The shRNA sequences targeting Rat Smad2, Smad3, CHSY1, c-jun and Sp1 were shown below: Smad2: 5'-GAT CTT CAA CAA CCA AGA ATT-3', Smad3: 5'-GCA ACC TGA AGA TCT TCA ACA-3', CHSY1: 5'-GTG AGG TTC ATG GGA AAC TTT-3', c-Jun: 5'-GGC TAC AGT AAC CCT AAG ATT-3', Sp1: 5'-CCT TCA CAA CTC AAG CTA TTT-3', and Control shRNA: 5'-UUC UCC GAA CGU GUC ACG UTT-3'. The backbone vector for shRNA was the GV248 vector (GeneChem, Shanghai, China). Lentivirus was produced by cotransfecting the GV248 vector, 
pHelper 1.0 and pHelper 2.0 packing plasmids (GeneChem) into 293T cells using Lipofectamine 2000 (Invitrogen). Supernatant was collected $48 \mathrm{~h}$ post transfection and virus was concentrated by centrifugation. Nucleus pulposus cells were then infected with lentivirus at a multiplicity of infection (MOI) 20.

\section{DMMB assay}

The proteoglycan content in the cells was measured as sGAG by colorimetric assay with 1,9-dimethylmethylene blue (DMMB) (Sigma) and chondroitin-4-sulfate as a standard following the manufacturer's instructions. Briefly, GAGs were precipitated from cell extracts and conditioned medium and stained with DMMB. Staining was quantified by measuring absorbance at $656 \mathrm{~nm}$. Results were calculated as GAG (mg)/total DNA (ng) and expressed relative to the value obtained from untreated controls.

\section{Statistical analysis}

Data represent mean \pm SD of at least 3 independent experiments with 3 replicates per experiment. Student t-test was used to analyze the significance between the two groups. ANOVA (one-way analysis of variance) with Bonferroni post-hoc test was used for the comparison of data from more than two groups. $P$ $<0.05$ was considered to indicate a statistically significant result.

\section{Results}

TGF- $\beta$ induces CHSY1 expression in rat NP cells

It has been reported that TGF- $\beta$ is able to induce biosynthesis of chondroitin sulfate proteoglycans in NP cells. CHSY1 is the key enzyme involved in the elongation of the carbohydrate side chain of the proteoglycans. To determine the expression of CHSY1, we examined the expression of CHSY1 in rat NP cells with stimulation of TGF- $\beta$. The expression of CHSY1 in NP cells was elevated significantly in response to TGF- $\beta$ treatment at both mRNA and protein levels. The initial of the induction was observed starting from $4 \mathrm{~h}$ post treatment and the maximum expression was observed $24 \mathrm{~h}$ after TGF- $\beta$ treatment (Fig. 1A and B). Additionally, the induction of CHSY1 by TGF- $\beta$ was in a concentration-dependent manner, as both CHSY1 mRNA and protein levels were elevated with the increase of TGF- $\beta$ concentration (Fig. 1C and D). The induction of CHSY1 by TGF- $\beta$ by immunofluorescent staining further confirmed the cytoplasmic localization of CHSY1 (Fig. 1E). Furthermore, the luciferase assay result showed that the CHSY1 promoter activity was highly enhanced upon TGF- $\beta$ treatment (Fig. $1 \mathrm{~F}$ ), indicating that the induction of CHSY1 by TGF- $\beta$ was through transcriptional activation. Taken together, we found that TGF- $\beta$ was able to transcriptionally activate the expression of the glycosyltransferase CHSY1 in rat NP cells.

\section{Knockdown of CHSY1 by specific shRNA impairs TGF- $\beta$ induced GAG accumulation in NP} cells

As previous results indicated that TGF- $\beta$ was able to induce CHSY1 expression and that CHSY1 level also correlated with sGAG accumulation in NP cells. To determine whether CHSY1 induced by TGF- $\beta$ contributes to the production of GAG shRNA specific for CHSY1 was used to knockdown its expression in NP cells. Both mRNA and protein of CHSY1 were significantly reduced, as measured by real-time PCR and Western blot (Fig. 2A and B). sGAG secretion assay indicated that cells with control shRNA had dramatically elevated sGAG secretion upon TGF- $\beta$ treatment. However, cells with shRNA targeting CHSY1 showed a decreased basal level of sGAG secretion and also failed to enhance sGAG level in response to TGF- $\beta$ stimulation (Fig. 2C). These results confirmed that elevated expression of CHSY1 by TGF- $\beta$ is required for the accumulation of GAG in NP cells.

Smad signaling is activated by TGF- $\beta$ and involved in TGF- $\beta$-induced CHSY1 expression

Activation of TGF- $\beta$ is known to trigger both Smad-dependent and -independent signaling pathways. To test which pathway is involved in the CHSY1-induced effect from TGF- $\beta$ in NP cells, the activation of Smads was first probed upon TGF- $\beta$ treatment in NP cells. 


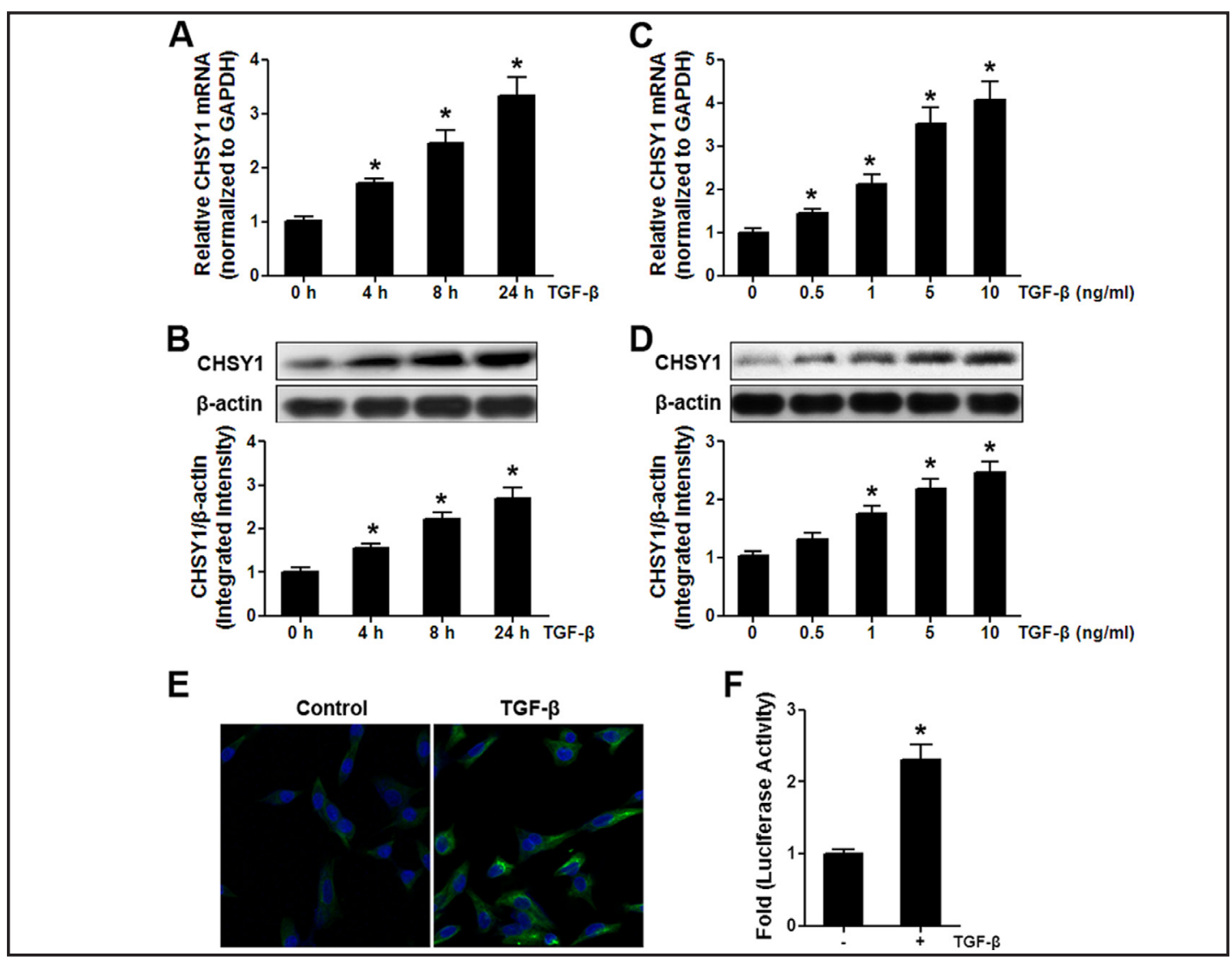

Fig. 1. TGF- $\beta$ increases CHSY1 expression in rat nucleus pulposus (NP) cells. RT-PCR analysis (A) and Western blot analysis (B) of CHSY1 expression by nucleus pulposus (NP) cells treated with TGF- $\beta$ (10 ng/ml) for 4, 8 and 24 hours. Values are mean $\pm \mathrm{SD} ; \mathrm{n}=3$. ${ }^{*} \mathrm{P}<0.05$ vs. control. RT-PCR analysis (C) and Western blot analysis (D) of CHSY1 expression by NP cells treated with TGF- $\beta$ for $24 \mathrm{~h}$ at indicated concentrations. Values are mean $\pm \mathrm{SD} ; \mathrm{n}=3 .{ }^{*} P<0.05$ vs. control. (E) Immunofluorescent analysis of CHSY1 in NP cells treated with TGF- $\beta$ for 24 h. (F) CHSY1 reporter activities measured following TGF- $\beta$ treatment. Values are mean \pm SD; $n$ $=3 .{ }^{*} P<0.05$ vs. control.

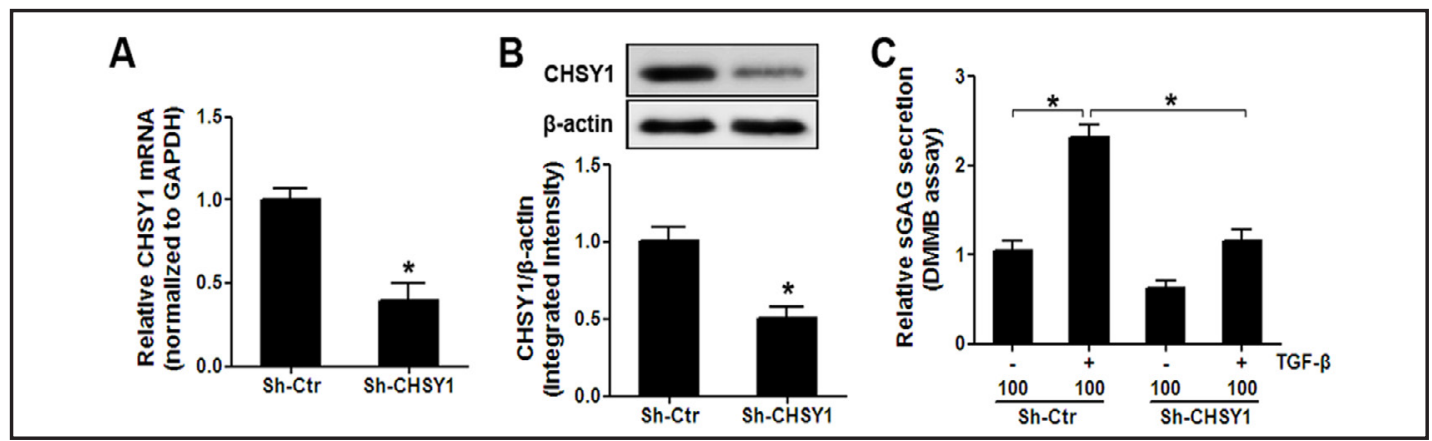

Fig. 2. Knockdown of CHSY1 by specific shRNA in NP cells decreases TGF- $\beta$ induced GAG accumulation. RT-PCR (A) and Western blot analysis (B) of rat NP cells transduced with control shRNA (Sh-Ctr) or short hairpin CHSY1 (Sh-CHSY1). Values are mean $\pm \mathrm{SD} ; \mathrm{n}=3 .{ }^{*} P<0.05$ vs. Sh-control. (C) GAG accumulation by nucleus pulposus cells treated with TGF- $\beta$ with or without shRNA for 5 days. Values are mean \pm SD; $n=4$. ${ }^{*} P<0.05$.

As shown in Fig. 3A, both Smad2 and Smad3 were quickly and significantly phosphorylated with the presence of TGF- $\beta$, confirming that the canonical Smad pathways were activated by TGF- $\beta$ in NP cells. To further examine, whether the activation of Smad pathways was KARGER 


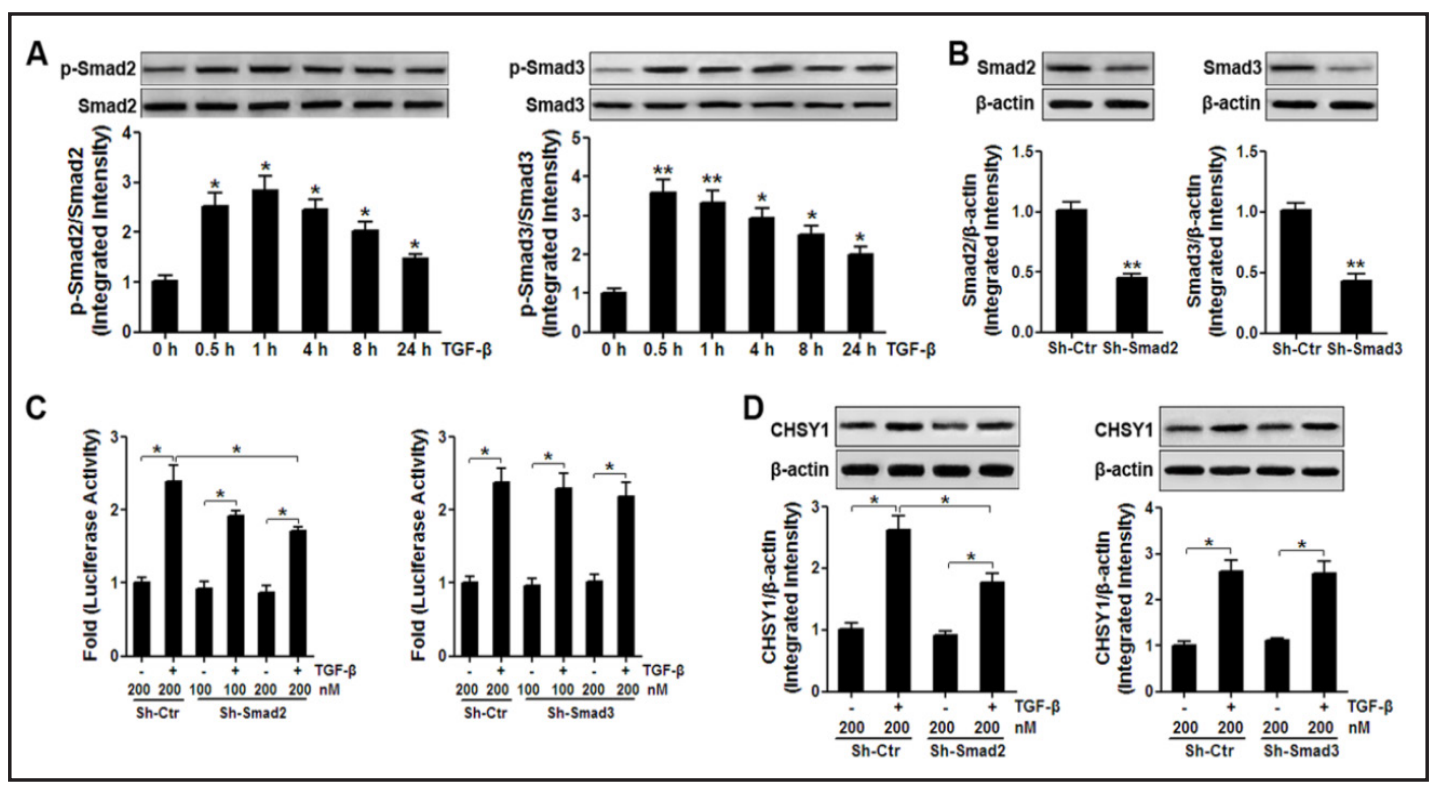

Fig. 3. Activated Smad signaling by TGF- $\beta$ is partially involved in TGF- $\beta$-induced CHSY1 expression in NP cells. (A) Western blot analysis of Smad 2 and Smad3 activation following TGF- $\beta$ treatment for the indicated times. Values are mean $\pm \mathrm{SD} ; \mathrm{n}=3 .{ }^{*} P<0.05,{ }^{* *} P<0.01$ vs. control. (B) Knockdown of Smad2 or Smad3 in NP cells. Knockdown of Smad2 in NP cells decreased CHSY1 reporter activity (C) and CHSY1 expression (D). Values in (B) are mean $\pm \mathrm{SD} ; \mathrm{n}=3 .{ }^{* *} P<0.01$ vs. Sh-control. Values in (C) and (D) are mean $\pm \mathrm{SD} ; \mathrm{n}=3 .{ }^{*} P<0.05$.

responsible for the induction of CHSY1, shRNA were used to knockdown the expression of Smad proteins. Significant reduction of both Smad2 and Smad3 levels was observed upon the shRNA transduction (Fig. 3B). The responsiveness of CHSY1 luciferase reporter to TGF- $\beta$ treatment was significantly blunted in Smad2 knockdown group instead of Smad3 knockdown group (Fig. 3C). Consistently, the induction of CHSY1 protein upon TGF- $\beta$ was inhibited in NP cells with Smad2 shRNA but not Smad3 shRNA, indicating that Smad2, but not Smad3, was involved in the regulation of CHSY1 expression. Taken together, these results suggested that the canonical Smad signaling pathways are activated by TGF- $\beta$ and partially involved in TGF- $\beta$-induced CHSY1 expression.

\section{AP1 and Sp1 activation is required for TGF- $\beta$-induced CHSY1 expression in NP cells}

To uncover the mechanism underlying TGF- $\beta$-induced CHSY1 expression in NP cells, the activation of known downstream effectors of the TGFpathway were tested, including AP1 and Sp1 transcription factors. Upon stimulation of TGF- $\beta$, phosphorylation of c-Jun, an indicator of active AP1, was greatly increased. The phosphorylation reached a plateau at $4 \mathrm{~h}$ post treatment and then slightly declined in $8 \mathrm{~h}$ and $24 \mathrm{~h}$, but remained significantly higher than basal level at $24 \mathrm{~h}$ after treatment (Fig. 4A). The expression of Sp1 transcription factor in response to TGF- $\beta$ showed a similar dynamic (Fig. 4A). Indeed, the luciferase assay showed that both AP1 and Sp1 transcription factors were active in TGF-treated NP cells (Fig. 4B), suggesting these factors are the mediators for TGF- $\beta$-induced CHSY1 expression. To test their correlation, lentivirus-mediated shRNAs were employed to knockdown c-Jun and Sp1 in NP cells (Fig. 4C). The promoter activity of CHSY1 in the presence of TGFwas dramatically suppressed when Sp1 or c-Jun were knockdown (Fig. 4D). As expected, TGF- $\beta$ stimulation elevated CHSY1 level; however, knockdown of c-Jun or Sp1 in NP cells significantly blunted the induction of CHSY1 by TGF- $\beta$ (Fig. 4E). Consistently, TGF- $\beta$ triggered sGAG secretion in control cells. The knockdown of c-Jun or Sp1 significantly attenuated sGAG secretion in the presence of TGF- $\beta$ compared with control group (Fig. 4F). In summary, our results indicated that AP1 and Sp1 activation is required for TGF- $\beta$-induced CHSY1 expression in NP cells.

\section{KARGER}




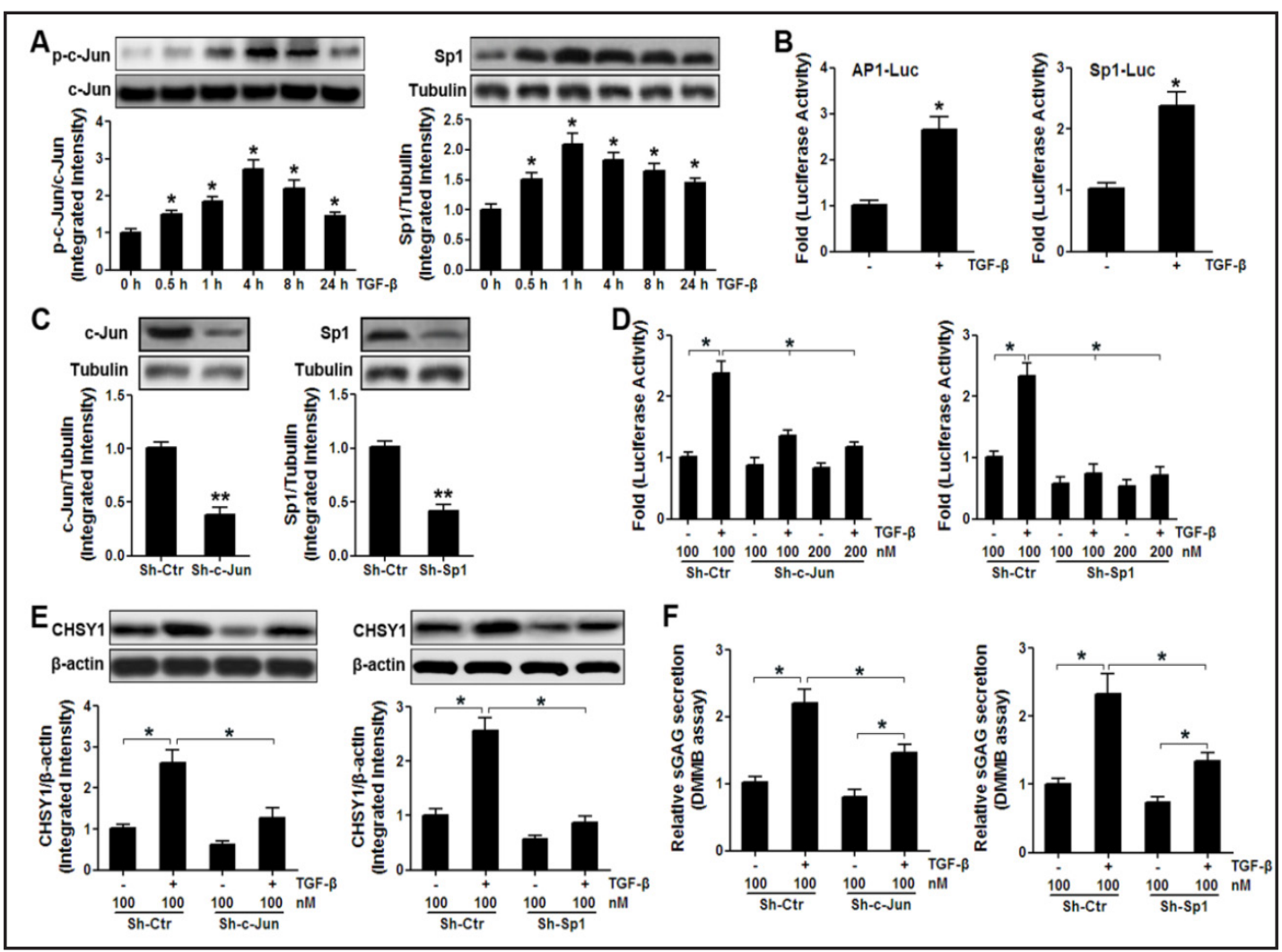

Fig. 4. AP1 and Sp1 activation is required for TGF- $\beta$-induced Chsy1 expression. (A) Western blot analysis of c-Jun and Sp1 following TGF- $\beta$ treatment for the indicated times. Values are mean \pm SD; $\mathrm{n}=3 .{ }^{*} P<0.05$ vs. control. (B) AP1 and Sp1 activities were determined by reporter assay following TGF- $\beta$ treatment for $24 \mathrm{~h}$. Values are mean $\pm \mathrm{SD} ; \mathrm{n}=3 .{ }^{*} P<0.05$ vs. control. (C) Knockdown of c-Jun or Sp1 in NP cells. Values are mean $\pm \mathrm{SD} ; \mathrm{n}=3 .{ }^{* *} \mathrm{P}<0.01$ vs. Sh-control. Knockdown of c-Jun or Sp1 in NP cells decreased CHSY1 reporter activity (D), CHSY1 expression (E) and GAG secretion (F). Values in (D) and (E) are mean $\pm \mathrm{SD} ; \mathrm{n}=3 .{ }^{*} P<0.05$. Values in $(\mathrm{F})$ are mean $\pm \mathrm{SD} ; \mathrm{n}=4 .{ }^{*} \mathrm{P}<0.05$.

\section{TGF- $\beta$ induced AP1 and Sp1 activation is regulated by MAPK signaling}

To further delineate the intercellular signaling cascades between TGF- $\beta$ treatment and activation ofAP1andSp1, theactivity ofMAPKpathwayswas probed.Interestingly, allthethreemajor MAPKsignaling pathways, includingERK, p38and JNK, were drastically activated upon TGFtreatment in NP cells (Fig. 5A). When treated together with SB431542, a specific inhibitor of TGF- $\beta$ receptor kinase, the increase of phosphorylation of ERK1/2, p38 and JNK from TGF- $\beta$ treatment was can significantly blunted (Fig. 5B). This result indicated that the activation of all these pathways were indeed a consequence of TGF- $\beta$. The data suggested that these signaling pathways could be essential for the regulation of CHSY1 expression, because the inhibition of all the MAPK activation by SB431542 not only contributed to the impaired induction of CHSY1 by TGF- $\beta$ (Fig. 5C), but also resulted in a partial reduction in CHSY1 promoter activity otherwise increased by TGF- $\beta$ (Fig. 5D). To further dissect whether individual MAPK pathway affects the TGF- $\beta$-induced responses of transcription factors AP1 and Sp1 in the regulation of CHSY1, we took advantage of chemical inhibitors specific for each pathway including PD98059 (PD) for ERK1/2, SP600125 (SP) for JNK, and SKF86002 (SKF) for p38. Strikingly, treatment of individual MAPK inhibitors completely abolished the transcriptional activation of both factors, AP1 and Sp1 (Fig. 5E and F), as well as decreased the CHSY1 promoter activity (Fig. 5 G) after TGF- $\beta$ treatment. Therefore, the results indicated that MAPK signaling pathways are the mediators required for TGF- $\beta$-induced AP1 and Sp1 activation in NP cells. 


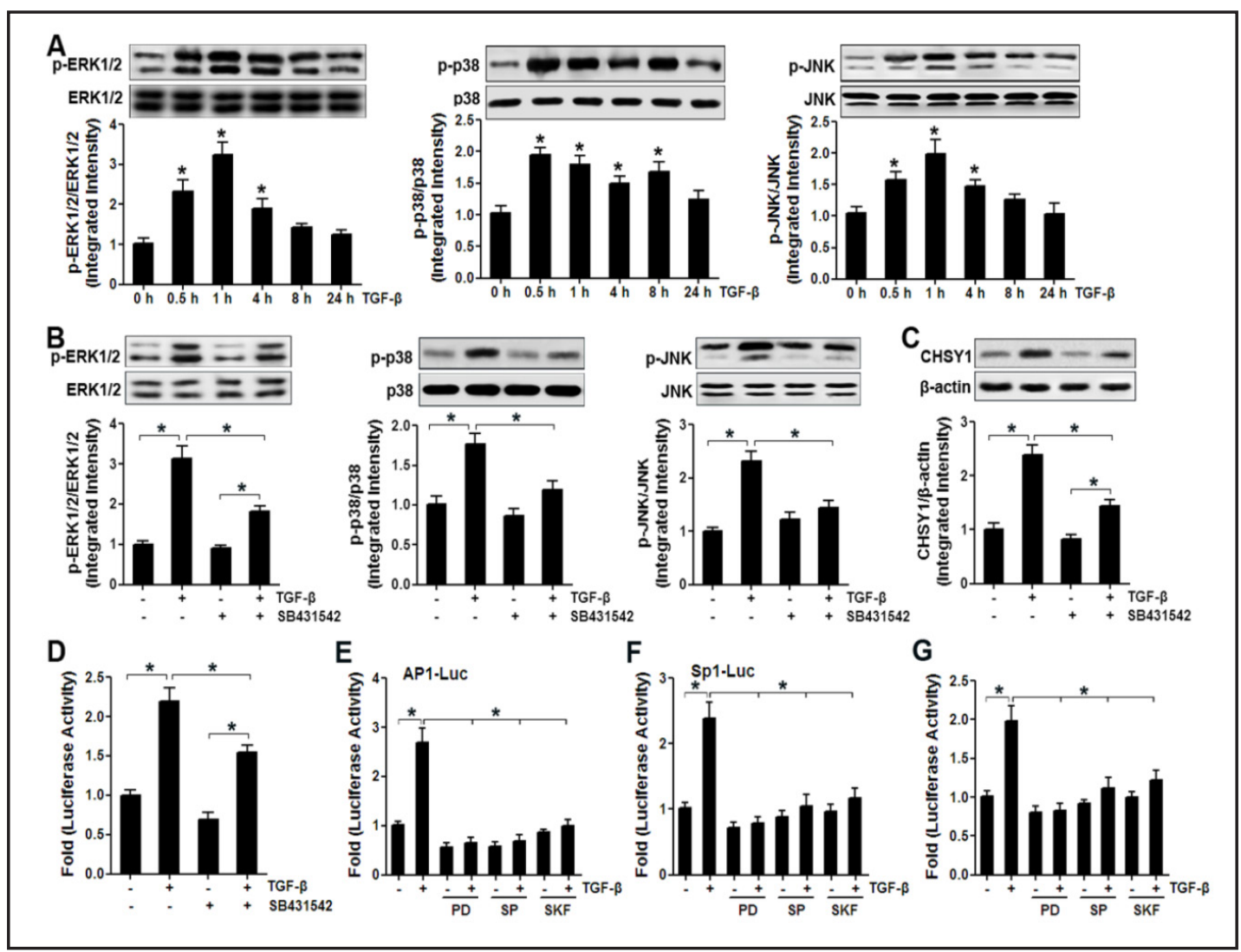

Fig. 5. MAPK pathway is required for TGF- $\beta$-induced AP1 and Sp1 activation in NP cells. (A) Western blot analysis of ERK1/2, p38 and JNK activation, following the treatment of nucleus pulposus cells with TGF- $\beta$ for the indicated times. Values are mean $\pm \mathrm{SD} ; \mathrm{n}=3 .{ }^{*} \mathrm{P}<0.05$ vs. control. (B) Western blot analysis of MAPKs following NP cells were treated with TGF- $\beta$ with or without SB431542 $(10 \mu \mathrm{M})$ for $1 \mathrm{~h}$. Values are mean \pm SD; $\mathrm{n}=3 .{ }^{*} \mathrm{P}<0.05$ vs. control. (C) CHSY1 expression measured following NP cells were treated with TGF- $\beta$ with or without SB431542 $(10 \mu \mathrm{M})$ for $24 \mathrm{~h}$. Values are mean $\pm \mathrm{SD} ; \mathrm{n}=3 .{ }^{*} P<0.05$. (D) CHSY1 reporter activities measured following NP cells were treated with TGF- $\beta$ with or without SB431542 (10 M) for 24 h. Values are mean $\pm \mathrm{SD} ; \mathrm{n}=3 .{ }^{*} P<0.05$. Nucleus pulposus cells were transfected with AP1, Sp1, or CHSY1 reporter plasmid. Transfected cells were treated with TGF- $\beta$ with or without MAPK inhibitors PD98059 (PD, $30 \mu \mathrm{M}$ ), SP600125 (SP, $30 \mu \mathrm{M}$ ), and SKF86002 (SKF, $20 \mu \mathrm{M}$ ) for $24 \mathrm{~h}$, and luciferase activity was measured. Effect of the treatment with inhibitors on AP1 (E), Sp1 (F), and CHSY1 reporter activity (G) was analyzed. Values in (E-G) are mean $\pm \mathrm{SD} ; \mathrm{n}=3 .{ }^{*} \mathrm{P}<0.05$.

\section{MAPK signaling is involved in CHSY1 expression by TGF- $\beta$}

It has been demonstrated that MAPK signaling pathways instigated by TGF- $\beta$ triggers the activation of AP1 and Sp1 transcription factors. To examine whether these signaling pathways are involved in the regulation of CHSY1 expression, NP cells were treated with inhibitors specific for individual MAPK pathways. Results showed that CHSY1 expression in the inhibitor-treated cells was no longer responsive to TGF- $\beta$ stimulation, with the greatest effect observed in the ERK1/2 inhibitor supplemented group (Fig. 6A). Immunofluorescent staining data further confirmed the failure of TGF- $\beta$ responsiveness in the MAPK inhibitor-treated cells. As shown in Fig. 6B, TGF- $\beta$ significantly induced CHSY1 expression in the cytoplasm of NP cells. However, the induction of CHSY1 was significantly decreased in all the PD, SP and SKF treated cells, suggesting the requirement of these MAPK signaling pathways in the regulation of CHSY1 expression with TGFtreatment. More importantly, the expression of CHSY1 reduced by MAPK inhibitors correlated well with the secretion of GAG in NP cells (Fig. 6C), implying the functional involvement of MAPK signaling in the biosynthesis of proteoglycans. 

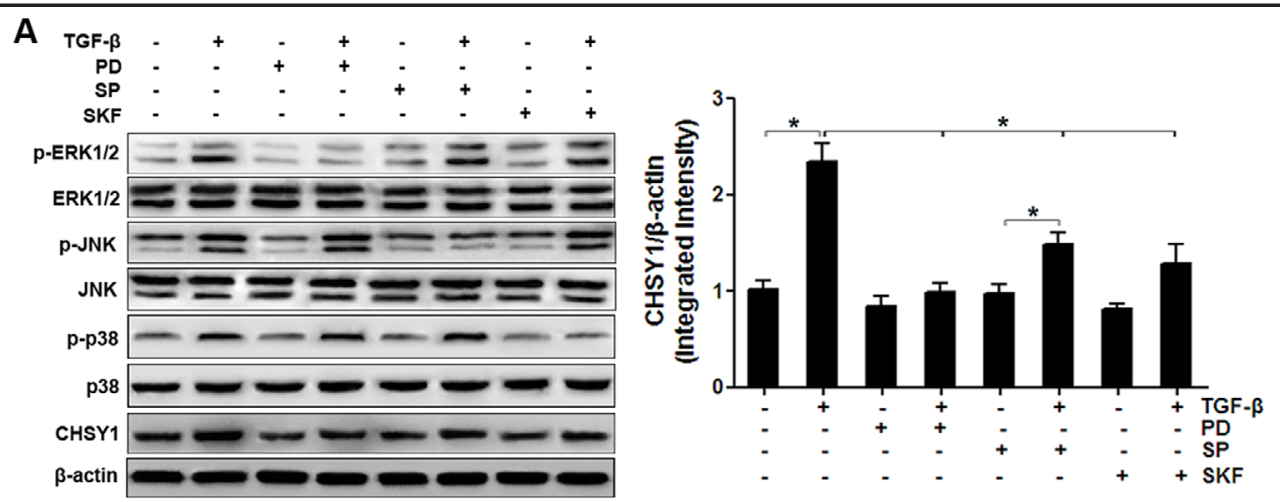

B
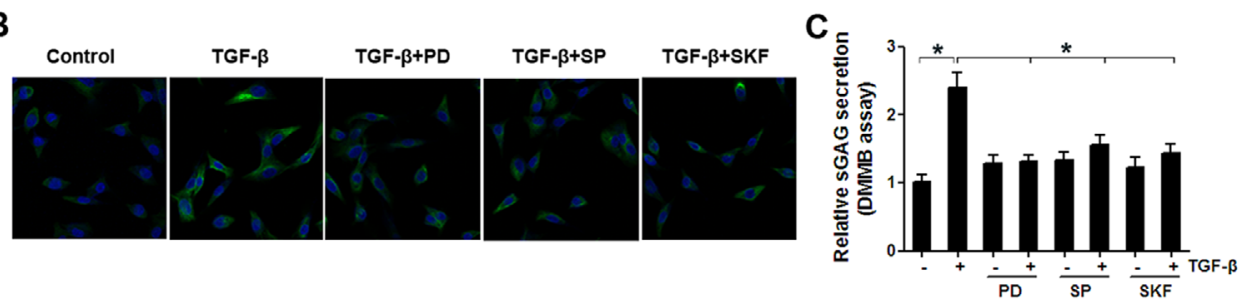

Fig. 6. MAPK regulation of CHSY1 expression in NP cells. (A) NP cells treated with TGF- $\beta$ with or without specific MAPK inhibitors PD98059 (PD, $30 \mu \mathrm{M}$ ), SP600125 (SP, $30 \mu \mathrm{M}$ ), and SKF86002 (SKF, $20 \mu \mathrm{M}$ ) for 4 h. Values are mean $\pm S D ; n=3 .{ }^{*} P<0.05$. (B) Immunofluorescent analysis of CHSY1 in NP cells treated with TGF- $\beta$ with or without MAPK inhibitors PD98059 (PD, $30 \mu \mathrm{M}$ ), SP600125 (SP, $30 \mu \mathrm{M}$ ), and SKF86002 (SKF, $20 \mu \mathrm{M}$ ) for $24 \mathrm{~h}$. (C) GAG accumulation by nucleus pulposus cells treated with TGF- with or without MAPK inhibitors PD98059 (PD, $30 \mu \mathrm{M}$ ), SP600125 (SP, $30 \mu \mathrm{M}$ ), and SKF86002 (SKF, $20 \mu \mathrm{M}$ ) for 5 days. Values are mean $\pm \mathrm{SD} ; \mathrm{n}=4 .{ }^{*} \mathrm{P}<0.05$.

\section{Discussion}

The nucleus pulposus represents one of the major components in the intervertebral discs. Dehydration reduced concentration of proteoglycans or degradation of extracellular matrix, which is the leading cause of disc degeneration [15-17]. It has been reported earlier that TGF- $\beta$ is able to induce the synthesis of GAG [13], a large carbohydrate side chains subunit of the important nucleus pulposus proteoglycan aggregan. GAG contributes to the maintenance of functional nucleus pulposus. However, the underlying mechanism for this beneficial effect of TGF- $\beta$ remains unknown. Herein, we demonstrated that TGF- $\beta$ induced the expression of CHSY1, which is a glycosyltransferase responsible for the formation of carbohydrate side chain of proteoglycans, in NP cells through the stimulation of MAPK pathways and subsequently the activation of AP1 and Sp1 transcription factors.

TGF- $\beta$ is a cytokine secreted by many cell types and is involved in a variety of different cellular processes $[18,19]$. It functions through binding to transmembrane serine/threonine protein kinase receptors to phosphorylate downstream targets. Smad transcription factor is one of the most canonical targets $[20,21]$. Additionally, ligand-bound TGF- $\beta$ receptors are also able to activate other pathways independent of Smad, such as ERK, p38, JNK MAPK and PI3K/AKT signaling pathways, as well as NF- $\kappa B$ and Rho GTPase [22-24]. The role of TGF- $\beta$ in the functionality of nucleus pulposus has been well appreciated. For example, TGF- $\beta 3$, a TGF- $\beta$ isoform, can support NP cell viability and promote phenotypic matrix deposition, thanks to the elevated GAG and type II collagen contents [12].

Interestingly, TGF- $\beta$ is known to trigger the expression of enzymes indispensible for the biosynthesis of proteoglycans in various tissues, such as CHSY1 in astrocytes upon injury [10], and beta1,3-glucuronosyl transferase 1 (GlcAT-1), in NP cells [25]. GlcAT-1 is an enzyme catalyzes the transfer of glucuronic acid (GlcUA) to the core protein Gal-GalXyl-O-Ser trisaccharide, leading to the initial branching of chondroitin sulfate [26]. On the KARGER 
other hand, CHSY1 possesses dual glucuronyl-transferase and galactosaminyl-transferase activity. It can promote the transfer of both GlcUA and N-acetylgalactosamine (GalNAc) from their donors UDP-GlcUA and UDP-GalNAc, respectively, to the non-reducing end of the chondroitin polymer, resulting in the elongating and accumulation of GAG [27]. Therefore, GlcAT-1 initiates the branching of chondroitin sulfate from the core protein, which provides the docking sites for CHSY1 to elongate the chondroitin [28]. It is likely that TGF- $\beta$ regulates key enzymes in proteoglycan metabolism in NP cells so as to ameliorate the functionality of NP tissue. In this present study, we found that rat NP cells treated with TGF- $\beta$ upregulated the glycosyltransferase, CHSY1, in a time and dose dependent manner. CHSY1 was essential for the assembly of proteoglycans in NP cells. Knockdown of CHSY1 led to reduced level of GAG in rested cells and decreased sensitivity to TGF- $\beta$ stimulation. The effects of TGF- $\beta$ on CHSY1 induction in NP cells was partially through the canonical Smad signaling pathway. A quick, strong and long-lasting Smad2 and Smad3 activation was observed upon TGF- $\beta$ treatment. When endogenous Smad2 were depleted, the induction of CHSY1 by TGF- $\beta$ was blunted. The induction of CHSY1 was partially transcriptional, because the CHSY1 promoter activity was significantly enhanced upon TGF- $\beta$ treatment. This transcriptional regulation of CHSY1 suggested the possibility that certain transcription factors were activated by TGF- $\beta$ in NP cells.

Among other transcription factors targeted by the TGF- $\beta$ pathway, AP1 and Sp1 factors are the known targets in NP cells closely related to TGF- $\beta$-induced gene expression in this particular cell type $[19,20]$. AP1 refers to a collection of dimeric transcription factors composed of proteins belonging to the c-Fos, c-Jun, ATF and JDP families. Sp1 is a zinc finger transcription factor that binds to GC-rich motifs in the promoters of a variety of many different genes [29]. In our study, both the expression and activity of AP1 and Sp1 were elevated in NP cells after treatment of TGF- $\beta$. Although the direct binding of these transcription factors to the CHSY1 promoter has not been tested, our knockdown experiments clearly indicated that both AP1 and Sp1 were indispensible for the induction of CHSY1 as well as the accumulation of GAG by TGF- $\beta$.

Given the facts that robust phosphorylation of the transactivation domain of AP1 was detected upon TGF- $\beta$ stimulation, and that AP1 is known to be phosphorylated by MAPK signaling pathways, it remains to be elucidated whether activated MAPK pathways by TGF- $\beta$ contribute to the activation of transcriptional machinery. As mentioned earlier, MAPK pathways represent one branch of the downstream signaling network of TGF- $\beta$. Indeed, all the three MAPK branches including ERK, p38 and JNK were dramatically activated by treatment with TGF- $\beta$. When the activation of MAPK pathways was blocked by chemical inhibitors, the transcription activity of AP1 and Sp1 was decreased. Furthermore, the promoter activity of CHSY1 was reduced and protein level of CHSY1 was blunted under the TGF- $\beta$ treatment. The treatment with MAPK inhibitors consistent with the treatment of TGF- $\beta$ receptor antagonist highlights the linear relation between TGF- $\beta$ and MAPK pathways. Although the literature has shown that ERK1 but not ERK2 is responsible for growth factor-mediated induction of GlcAT-1 and GAG accumulation in NP cells, our observation indicated, that not only ERK but also p38 and JNK were activated upon TGF- $\beta$ treatment. These data implied that all the MAPK pathways might be important for the effects of TGF- $\beta$ in these cells.

As our results indicated that activation of both Smad2 and MAPK pathways is required for the full induction of CHSY1 and subsequent accumulation of GAG, it is likely that these two branches of the TGF- $\beta$ signaling pathways work synergistically in NP cells. As mentioned earlier, activation of the TGF- $\beta$ receptors can not only directly trigger nuclear translocation of Smad2 and Smad3 but also initiate all ERK, p38 and JNK MAPKs [19]. The interaction between Smads and MAPKs is complicated and also cell-type dependent [30]. For example, Smad-dependent transcription responses have been shown to be responsible for some TGF$\beta$-induced MAPKs activation [30]; while on the other hand, MAPKs activation is also known to involve in Smad-mediated transcriptional regulation. It has been shown that MAPKs can directly phosphorylate Smad proteins, thereby alternating their cellular localization [31]. Additionally, MAPK-activated transcription factors, including AP1 and Sp1, are known to 
participate in the Smad complexes bound to DNA sequences with either Smad-binding site or AP1/Sp1 binding site [32]. As we shown that Smad2 activity is involved in the transcription of CHSY1; while in the presence of MAPK inhibitors, we observed suppression, rather than activation, of CHSY1, therefore, it is not likely that MAPKs negatively regulate Smad in this context. However, whether MAPKs phosphorylate Smad2 in NP cells in response to TGF- $\beta$ is still unknown. Furthermore, although we have not experimentally tested how the cooperation between Smad2 and MAPKs occurs in terms of CHSY1 regulation, it is likely that MAPK-activated AP1 and Sp1 selectively interact with the nuclear translocated Smad2 and direct Smad2 to the promoter region of the CHSY1 locus, resulting in transcriptional activation of this gene.

In conclusion, we demonstrated that enzyme CHSY1, which was essential for the biosynthesis of GAG in NP cells, was transcriptionally induced by the MAPK-AP1/Sp1 axis in response to TGF- $\beta$.

\section{Acknowledgements}

The study was funded by the Natural Science Foundation of China (No. 81171752) and Shanghai Health System Important Disease Joint Research Project (2013ZYJB0502).

\section{Disclosure Statement}

The authors stated that they had no interests, which might be perceived as posing a conflict.

\section{References}

1 Pattappa G, Li Z, Peroglio M, Wismer N, Alini M, Grad S: Diversity of intervertebral disc cells: phenotype and function. J Anat 2012;221:480-496.

2 Horner HA, Roberts S, Bielby RC, Menage J, Evans H, Urban JP: Cells from different regions of the intervertebral disc: effect of culture system on matrix expression and cell phenotype. Spine (Phila Pa 1976) 2002;27:1018-1028.

3 Setton LA, Chen J: Mechanobiology of the intervertebral disc and relevance to disc degeneration. J Bone Joint Surg Am 2006;88:52-57.

4 Papadimitriou A, Peixoto EB, Silva KC, Lopes de Faria JM, Lopes de Faria JB: Inactivation of AMPK mediates high phosphate-induced extracellular matrix accumulation via NOX4/TGFss-1 signaling in human mesangial cells. Cell Physiol Biochem 2014;34:1260-1272.

5 Nap RJ, Szleifer I: Structure and interactions of aggrecans: statistical thermodynamic approach. Biophys J 2008;95:4570-4583.

6 Wilson DG, Phamluong K, Lin WY, Barck K, Carano RA, Diehl L, Peterson AS, Martin F, Solloway MJ: Chondroitin sulfate synthase 1 (Chsy1) is required for bone development and digit patterning. Dev Biol 2012;363:413-425.

7 Li Y, Laue K, Temtamy S, Aglan M, Kotan LD, Yigit G, Canan H, Pawlik B, Nürnberg G, Wakeling EL, Quarrell OW, Baessmann I, Lanktree MB, Yilmaz M,Hegele RA, Amr K, May KW, Nürnberg P, Topaloglu AK, Hammerschmidt M, Wollnik B: Temtamy preaxial brachydactyly syndrome is caused by loss-of-function mutations in chondroitin synthase 1, a potential target of BMP signaling. Am J Hum Genet 2010;87:757767.

8 Tian J, Ling L, Shboul M, Lee H, O'Connor B, Merriman B, Nelson SF, Cool S, Ababneh OH, Al-Hadidy A, Masri A, Hamamy H, Reversade B: Loss of CHSY1, a secreted FRINGE enzyme, causes syndromic brachydactyly in humans via increased NOTCH signaling. Am J Hum Genet 2010;87:768-778.

9 Sakai K, Kimata K, Sato T, Gotoh M, Narimatsu H, Shinomiya K, Watanabe H: Chondroitin sulfate $\mathrm{N}$-acetylgalactosaminyltransferase-1 plays a critical role in chondroitin sulfate synthesis in cartilage. J Biol Chem 2007;282:4152-4161. 
10 Yang T, Chen M, Sun T: Simvastatin attenuates TGF-beta1-induced epithelial-mesenchymal transition in human alveolar epithelial cells. Cell Physiol Biochem 2013;31:863-874.

11 Liu D, Cui W, Liu B, Hu H, Liu J, Xie R, Yang X, Gu G, Zhang J, Zheng H: Atorvastatin protects vascular smooth muscle cells from TGF-beta1-stimulated calcification by inducing autophagy via suppression of the betacatenin pathway. Cell Physiol Biochem 2014;33:129-141.

12 Risbud MV, Di Martino A, Guttapalli A, Seghatoleslami R, Denaro V, Vaccaro AR, Albert TJ, Shapiro IM: Toward an optimum system for intervertebral disc organ culture: TGF-beta 3 enhances nucleus pulposus and anulus fibrosus survival and function through modulation of TGF-beta-R expression and ERK signaling. Spine (Phila Pa 1976) 2006;31:884-890.

13 Susarla BT, Laing ED, Yu P, Katagiri Y, Geller HM, Symes AJ: Smad proteins differentially regulate transforming growth factor-beta-mediated induction of chondroitin sulfate proteoglycans. J Neurochem 2011;119:868-878.

14 Risbud MV, Guttapalli A, Stokes DG, Hawkins D, Danielson KG, Schaer TP, Albert TJ, Shapiro IM: Nucleus pulposus cells express HIF-1 alpha under normoxic culture conditions: a metabolic adaptation to the intervertebral disc microenvironment. J Cell Biochem 2006;98:152-159.

15 Chen JW, Ni BB, Li B, Yang YH, Jiang SD, Jiang LS: The responses of autophagy and apoptosis to oxidative stress in nucleus pulposus cells: implications for disc degeneration. Cell Physiol Biochem 2014;34:11751189.

16 Foley CJ, Luo C, O'Callaghan K, Hinds PW, Covic L, Kuliopulos A: Matrix metalloprotease-1a promotes tumorigenesis and metastasis. J Biol Chem 2012;287:24330-24338.

17 Choi H, Johnson ZI, Risbud MV: Understanding Nucleus Pulposus Cell Phenotype: A Prerequisite for Stem Cell Based Therapies to Treat Intervertebral Disc Degeneration. Curr Stem Cell Res Ther 2015;10:307-316.

18 Teng Y, Zhao L, Zhang Y, Chen W, Li X: Id-1, a protein repressed by miR-29b, facilitates the TGFbeta1induced epithelial-mesenchymal transition in human ovarian cancer cells. Cell Physiol Biochem 2014;33:717-730.

19 Shi Y, Massague J: Mechanisms of TGF-beta signaling from cell membrane to the nucleus. Cell 2003;113:685-700.

20 Kang JS, Liu C, Derynck R: New regulatory mechanisms of TGF-beta receptor function. Trends Cell Biol 2009;19:385-394.

21 Moustakas A, Heldin CH: The regulation of TGFbeta signal transduction. Development 2009;136:36993714.

22 Moustakas A, Heldin CH: Non-Smad TGF-beta signals. J Cell Sci 2005;118: 3573-3584.

23 Zhang YE: Non-Smad pathways in TGF-beta signaling. Cell Res 2009;19:128-139.

24 Luo C, Sheng J, Hu MG, Haluska FG, Cui R, Xu Z, Tsichlis PN, Hu GF, Hinds PW: Loss of ARF sensitizes transgenic BRAFV600E mice to UV-induced melanoma via suppression of XPC. Cancer Res 2013;73:43374348.

25 Hiyama A, Gogate SS, Gajghate S, Mochida J, Shapiro IM, Risbud MV: BMP-2 and TGF-beta stimulate expression of beta1,3-glucuronosyl transferase 1 (GlcAT-1) in nucleus pulposus cells through AP1, TonEBP, and Sp1: role of MAPKs. J Bone Miner Res 2009;25:1179-1190.

26 Pedersen LC, Tsuchida K, Kitagawa H, Sugahara K, Darden TA, Negishi M: Heparan/chondroitin sulfate biosynthesis. Structure and mechanism of human glucuronyltransferase I. J Biol Chem 2000;275:3458034585.

27 Kitagawa H, Uyama T, Sugahara K: Molecular cloning and expression of a human chondroitin synthase. J Biol Chem 2001;276:38721-38726.

28 Kawashima C, Terayama K, Ii M, Oka S, Kawasaki T: Characterization of a glucuronyltransferase: neolactotetraosylceramide glucuronyltransferase from rat brain. Glycoconj J 1992;9:307-314.

29 Sheng J, Luo C, Jiang Y, Hinds PW, Xu Z, Hu GF: Transcription of angiogenin and ribonuclease 4 is regulated by RNA polymerase III elements and a CCCTC binding factor (CTCF)-dependent intragenic chromatin loop. J Biol Chem 2014;289:12520-12534.

30 Javelaud D, Mauviel A: Crosstalk mechanisms between the mitogen-activated protein kinase pathways and Smad signaling downstream of TGF-beta: implications for carcinogenesis. Oncogene 2005;24:5742-5750.

31 Kretzschmar M, Doody J, Timokhina I, Massague J: A mechanism of repression of TGFbeta/ Smad signaling by oncogenic Ras. Genes Dev 1999;13:804-816.

32 Zhang Y, Feng XH, Derynck R: Smad3 and Smad4 cooperate with c-Jun/c-Fos to mediate TGF-beta-induced transcription. Nature 1998;394:909-913. 